\title{
Decision fusion in healthcare and medicine: a narrative review
}

\author{
Elham Nazari ${ }^{1} \wedge$, Rizwana Biviji ${ }^{2} \wedge$, Danial Roshandel ${ }^{3} \wedge, \operatorname{Reza}_{\text {Pour }}{ }^{4}$, Mohammad Hasan Shahriari $^{5}$, \\ Amin Mehrabian ${ }^{6} \wedge$, Hamed Tabesh ${ }^{1 \wedge}$
}

${ }^{1}$ Department of Medical Informatics, Mashhad University of Medical Sciences, Mashhad, Iran; ${ }^{2}$ Science of Healthcare Delivery, College of Health Solutions, Arizona State University, Phoenix, AZ, USA; ${ }^{3}$ Centre for Ophthalmology and Visual Science (affiliated with the Lions Eye Institute), The University of Western Australia, Perth, Western Australia, Australia; ${ }^{4}$ Department of Computer Engineering, Azad University, Mashhad, Iran; ${ }^{5}$ Department of Health Information Technology and Management, School of Allied Medical Sciences, Shahid Beheshti University of Medical Sciences, Tehran, Iran; ${ }^{6}$ Warwick Medical School, University of Warwick, Coventry, UK

Contributions: (I) Conception and design: E Nazari, H Tabesh; (II) Administrative support: E Nazari, H Tabesh; (III) Provision of study materials or patients: E Nazari, H Tabesh; (IV) Collection and assembly of data: All authors; (V) Data analysis and interpretation: All authors; (VI) Manuscript writing: All authors; (VII) Final approval of manuscript: All authors.

Correspondence to: Rizwana Biviji, PhD, MS. College of Health Solutions, 550 North 3rd Street, Phoenix, AZ 85004, USA.

Email: Rizwana.Biviji@asu.edu; Hamed Tabesh, PhD. Department of Medical Informatics, Azadi Square, Mashhad University of Medical Sciences, Mashhad, Iran. Email: Tabeshh@mums.ac.Ir.

Objective: To provide an overview of the decision fusion (DF) technique and describe the applications of the technique in healthcare and medicine at prevention, diagnosis, treatment and administrative levels.

Background: The rapid development of technology over the past 20 years has led to an explosion in data growth in various industries, like healthcare. Big data analysis within the healthcare systems is essential for arriving to a value-based decision over a period of time. Diversity and uncertainty in big data analytics have made it impossible to analyze data by using conventional data mining techniques and thus alternative solutions are required. DF is a form of data fusion techniques that could increase the accuracy of diagnosis and facilitate interpretation, summarization and sharing of information.

Methods: We conducted a review of articles published between January 1980 and December 2020 from various databases such as Google Scholar, IEEE, PubMed, Science Direct, Scopus and web of science using the keywords decision fusion (DF), information fusion, healthcare, medicine and big data. A total of 141 articles were included in this narrative review.

Conclusions: Given the importance of big data analysis in reducing costs and improving the quality of healthcare; along with the potential role of DF in big data analysis, it is recommended to know the full potential of this technique including the advantages, challenges and applications of the technique before its use. Future studies should focus on describing the methodology and types of data used for its applications within the healthcare sector.

Keywords: Decision fusion (DF); information fusion; healthcare; medicine; big data

Received: 01 July 2021; Accepted: 02 August 2021; Published: 20 January 2022.

doi: $10.21037 /$ mhealth-21-15

View this article at: https://dx.doi.org/10.21037/mhealth-21-15

^ ORCID: Elham Nazari, 0000-0002-3169-0396; Rizwana Biviji, 0000-0002-3286-3332; Danial Roshandel, 0000-0002-6716-6406; Mohammad Hasan Shahriari, 0000-0001-5596-1958; Amin Mehrabian, 0000-0002-8879-7565; Hamed Tabesh, 0000-0003-3081-0488. 


\section{Introduction}

The production of large amounts of data has become inevitable in the last 20 years owing to the advent of various technologies such as computers, mobile phones and smart devices as well as the rapid development of the internet at an unprecedented rate $(1,2)$. Healthcare is one of the largest industries with a rapid data growth (1). In 2011, the healthcare data in the United States was as large as $150 \times 10^{18}$ bytes. The California Health Network believes its electronic health record (EHR) system contains $26.5 \times 10^{15}-44 \times 10^{15}$ bytes data (3). This type of data is known as "big data". By and large conclusions drawn from big data analytics alone are often insufficient and a rigorous small data paradigm that functions autonomously and collaboratively with big data analytics is considered essential (4). The big data approach refers to analyzing data that is collected from a set of individuals with the goal of predicting a phenomenon for a larger population while, the small data paradigm is used to achieve data predictions at an individual level (e.g., a person, clinic, or community) (4). As such the use of artificial intelligence and machine learning is more commonly linked with 'big data'. However, it is very important to manage and analyze this kind of data effectively to discover new knowledge, improve care and reduce costs (5-9). As an example, the process of obtaining a human genome data took approximately ten years in the past, while the process is currently performed in less than a week by using data analysis techniques (5). In addition, these analyses have been very useful in cases where there is a need for aggregation of different specializations and appropriate decisions for the diagnosis or treatment. For example, there have been vast improvements in diagnostic accuracy in the early diagnosis of cancers, which is partly attributed to latest advancements in techniques for big data analysis. Big data analysis has many advantages such as improved quality of health care services and cost savings, but there are certain disadvantages that need consideration. One of the biggest barriers for applying big data techniques in health care is the distributed nature of medical data. Data is stored by different providers, such as insurers, contingent on the city and country. Another challenge is the variety of data, which would require decision making under uncertainty and fuzziness. A promising approach to overcome these challenges is through the use of decision fusion (DF) technique that was first introduced in the literature in 1960s, as mathematical models for data manipulation (10). The terms DF, data fusion, information fusion and sensor fusion are often used synonymously in the literature (11). However, in certain instances the term data fusion is used for raw data and $\mathrm{DF}$ /information fusion is used to identify already processed data (11). DF is related to a combination of decisions from various entities and is known as a higher semantic level fusion task than data fusion techniques $(11,12)$.

The motivation behind using DF technique is to obtain data from multiple sources, thereby providing more meaningful information about the system under investigation compared to analyzing single data sources separately (13). Results from different algorithms, methods, sources or classifiers can be fused. The overarching goal of the DF technique is to reduce the complexity of data to a manageable level for a human analyst or operator and is used to optimize and summarize the information being presented $(7,14,15)$. This technique is related to data mining. It uses the concepts of various fields such as psychology, human factors, knowledge representation, artificial intelligence, mathematical logic and signal processing. The DF technique uses basic machine learning methods. In addition, it uses voting, weighting, competitive approaches and other approaches to determine the value of decisions to combine (16).

DF has been successfully applied in various fields including the healthcare with respect to recommender systems (17), image processing (18), signal processing (19), medical diagnostics (20), rehabilitation (21) and genetic analysis (22). DF has several advantages such as increasing the accuracy of diagnosis, ease of interpretation, and summarizing and sharing of the information $(5,6,23,24)$. This narrative review provides an overview of the DF technique and describes the applications of the technique in healthcare and medicine at prevention, diagnosis, treatment and administrative levels.

We present the following article in accordance with the Narrative Review reporting checklist (available at https:// dx.doi.org/10.21037/mhealth-21-15).

\section{Methods}

For this narrative review a comprehensive search was conducted on six bibliographic electronic databases (Google Scholar, IEEE, PubMed, Science Direct, Scopus and web of science) using the keywords decision fusion (DF), information fusion, symbolic fusion, distributed decisions, expert fusion, and sensor fusion in conjunction with med* and healthcare. We included all relevant literature (i.e., books, surveys, review papers and original research papers) 
published in the English language between January 1980 and December 2020 in accordance with the PRISMA guidelines (25). We included literature (I) written in English language, (II) implemented at least one DF technique (III) intended use in the fields of healthcare and medicine. Two reviewers (EN and HT) independently evaluated the records for inclusion. The initial search resulted in 2,560 citations. After review, 2,419 articles were excluded by one or more of the following criteria (I) duplicate article (II) did not meet the inclusion criteria (III) full text of the article was not available (IV) article was about proposing framework, commentary, viewpoint, proposal, protocol and editorial. At completion of review, 141 relevant articles were included for further discussion. We followed a 3-step process for data abstraction and categorization. First, we categorized the articles by the analytical domain. Next, we categorized it based on the four levels of care, i.e., prevention, diagnosis, treatment \& rehabilitation and administrative. Thereafter, we classified it based on the methodology employed.

A narrative synthesis was performed on results extracted from the set of studies included in this review. The remainder of this paper continues as follows. The Introduction Section provides various classification categories for DF techniques, discusses general applications, summarizes their advantages and describes the most common methods for data association tasks. The Method Section details the use of DF technique in the healthcare field, classifies its applications based on the four levels of healthcare delivery and further enumerates the use of this technique within each of these levels, i.e., prevention, diagnosis, treatment \& rehabilitation and administrative. Since this was a narrative review of literature, informed consent was not required.

\section{Narrative review}

\section{DF technique}

The DF system can be viewed as a special case of data fusion system (26), which is defined as "data fusion techniques combine data from multiple sensors and related information from associated databases to achieve improved accuracy and more specific inferences than could be achieved by the use of a single sensor alone" (27). Data fusion technique refers to fusing of data first followed by the detection and recognition of object within the fused data (26). On the other hand, DF technique focuses on data detection and recognition within objects in each signal or image separately before fusing the decisions (26). DF uses a set of classifiers to offer an improved and unbiased result. These classifiers can be of identical or different types and feature sets (16). There are multiple reasons of preferring a multiclassifier system such as DF over a single classifier (16), e.g., (I) a multiclassifier system is an efficient approach to handle large volume of data, where the data is partitioned into smaller subsets, trained with different classifiers for different subsets and the outputs are combined; (II) using multiple classifiers with a subset of features may enhance performance; (III) multiple classifiers can be combined to give a better output than a single classifier.

The multiclassifier system can be classified in several ways based on the methods and techniques employed (11). They can be classified based on the relation between data sources $(11,28)$, such as: (I) complementary, where information provided by input sources do not depend on each other, but can be combined to obtain a more complete global information. They assist in reducing the problem of incompleteness; (II) competitive, where multiple input sources provide information of the same target and the redundant information could be fused to increase confidence. The aim here is to reduce measurement uncertainty and erroneous measurements; (III) cooperative, where the information is combined into new information, which is typically more complex than the original information and eliminates ambiguities. They can also be classified based on abstraction levels $(11,29)$, such as: (I) signal level, which directly addresses the signals that are acquired from the sensors; (II) pixel level, which operates at the image level often used to improve image processing tasks; (III) characteristic level, which employs features that are extracted from images or signals. (IV) symbol level, where information is presented as symbols and is also known as decision level.

\section{Applications of DF}

The most important uses of the DF technique include preprocessing, building models, and extracting information. This technique can be used in diverse domains such as command and control, intelligence analysis, strategic analysis, counterterrorism and homeland security, economics and biology (12). Moreover, this technique can specifically be used in remote sensing, medical image processing, object identifying, speech processing, classifying, retrieving and detecting genes in DNA (Deoxyribonucleic Acid) sequences. Additionally, there are other applications, 
including hospital information system (HIS), EHR and decision support system (DSS) $(12,16,30,31)$.

\section{Advantages of $D F$}

The key advantages of the DF technique include: effective management of non-homogeneous data sources (32), efficient combination of data sources, reduction in dimensionality and certain noises (33), improved model performance $(34,35)$, ease of interpretation of results (36), clear display and summarization of information, ease of information extraction, improved decision-making and cost reductions. Other potential advantages include: decrease in the loss of information and meaningful use of information, which improves results, data quality, reliability and entity detection (37). Further, it reduces ambiguity and uncertainty $(38,39)$, and manages massive volumes of data or communications that are seemingly unrelated (15). It is also used for extraction of features that cannot be extracted by using individual resources (38). This technique allows the decision-maker to discover and explore behavioral patterns or activities (6) that contribute towards improving the capabilities of intelligent systems (38). However, it is important to address certain challenges such as low quality of data used for analysis, complexity of the data $(40,41)$, inappropriate method selection depending on the type of data, inconsistent and incomplete data (42) imbalanced data classification (43) and computational burden $(24,44)$.

\section{DF methods}

The methods used in the DF technique generally fall into three categories: (I) probabilistic method, (II) Evidential method, and (III) Intelligent method. Probabilistic methods use probability and statistical foundations. An example of this method is the Bayesian theory, which has been effectively used in fusion event probabilities and managing randomness in the DF technique. The Causal independence model (36), the Belief function theory method (45), the discrete Bayes classifier method (46), the Bayesian method (34,47-51) and the likelihood method (52) are all examples of probabilistic methods that have been used in various studies. The evidential method pertains to combining pieces of evidence to calculate the probability of an event. For instance, the Dempster-Shafer (DS) theory uses the belief attribute. The most important part of this theory is the Dempster's rule of combination, which combines evidence from two or more sources to produce an inference
$(33,40,53-57)$. DS theory is a generalization of the Bayesian theory of subjective probability. To clarify, Bayesian and DS theories deal with a kind of uncertainty $(33,40,53-57)$. These methods have been applied in several healthcare studies $(33,40,53-57)$. Investigators have used methods such as particle swarm optimization (PSO) $(35,58,59)$, fuzzy $(15,60)$, support vector machine (SVM) (61-64), decision tree (65), k-means (66-68), neural network (69), Markov (67), case base reasoning (CBR) (70) and K-Nearest neighbors algorithm (KN) (36), which are examples of the intelligent method. Fuzzy-logic theory has been used effectively in fuzziness information processing. Fuzzy methods are suitable for qualitative information, but they are weak in managing randomness. Advanced concepts, in this case, include fuzzy logic Type-2 (FIS2) and computing with words (CWW) that attempt to manage fuzziness and randomness in events (16). Recently, cloud computing theory (CCT) has been introduced as a computational methodology for combining randomness and fuzziness in the information used in decision making. Cloud theory also provides a method for modeling qualitative concepts and turning them into quantitative measures $(3,16,71)$. In other healthcare studies, conventional methods such as the Ensemble method have been used (72-75). Ensemble methods are efficient classical techniques that work based on weighting or voting and have been used in machine learning and described in most machine learning books (38). Furthermore, convolutional neural network (CNN) model (76) and CNN ensemble method (77) have been used in the healthcare area, out of which the latter is a mixed method. In addition, Deep learning methods (78) and the new algorithmic base method $(32,41,42,58,79-96)$ have also been applied in various healthcare fields. Software such as Microsoft C ++ $(83,86,97)$, MATLAB $(19,75,79,86,98-101), \mathrm{C} \#(84)$, Weka $(61,102)$ and R $(52,74)$ have been used to implement these methods.

DF techniques can be categorized into quantitative or qualitative methods. Quantitative methods include evidence theory, probability theory, fuzzy sets, random sets and possibility theory. Quantitative methods are the best means for displaying and managing uncertainty, while qualitative methods are preferred for reasoning and knowledge. Combined methods include quantitative logics, incidence and calculus. A good solution for global modeling in this situation can be a hybrid approach (7). Recently, Deep learning methods have been highly regarded for computational complexity issues. These methods have been used in various health care studies that will be discussed in 


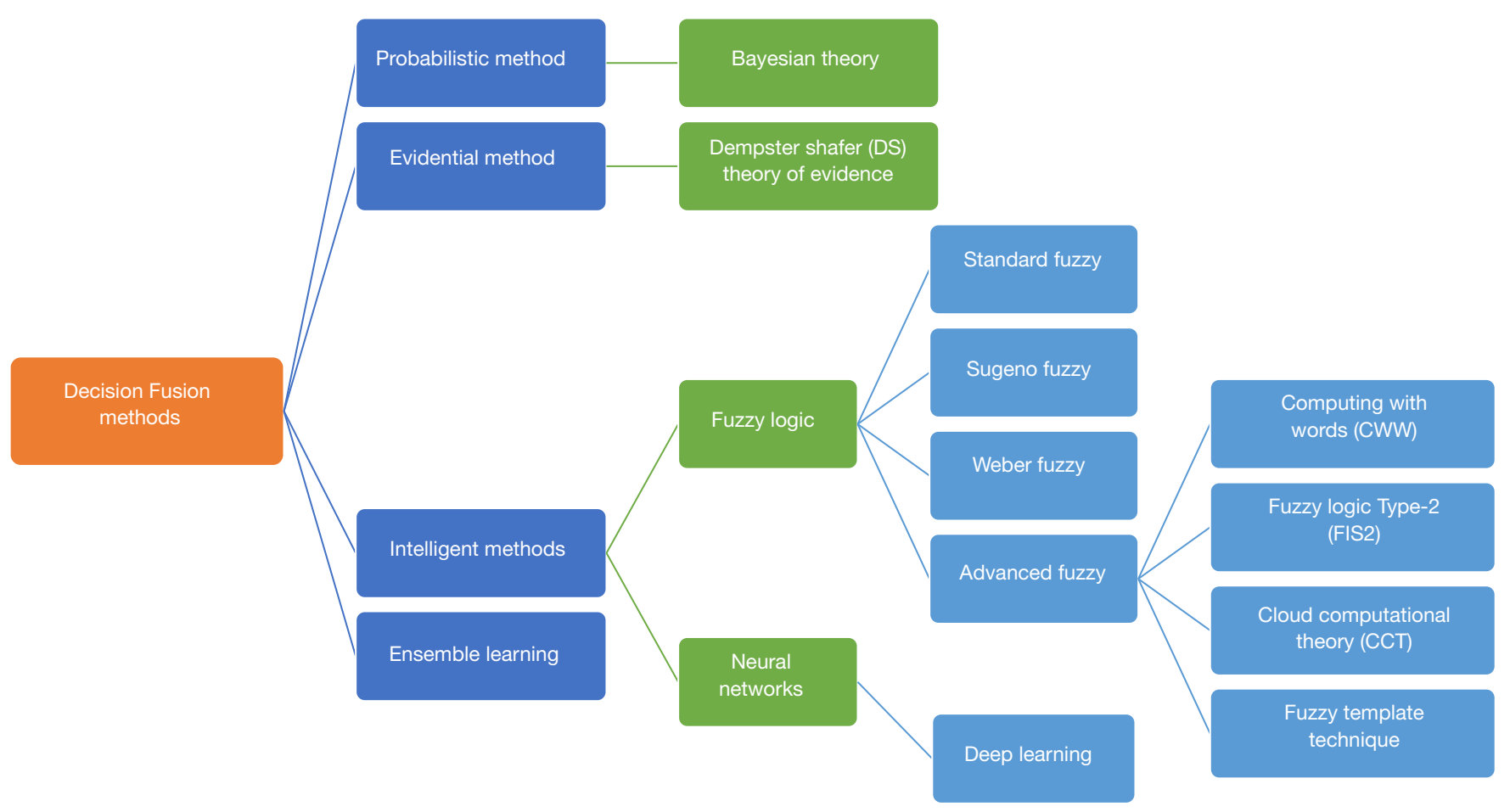

Figure 1 Types of methods used in the decision fusion technique.

the DF application section. Figure 1 provides a schematic representation of the types of methods used in the DF technique.

\section{DF in the bealthcare field}

The incidences of various chronic and infectious diseases are on the rise, which is evident through the spread of coronavirus in recent months. Interestingly, the DF technique is also applied in the field of research related to COVID-19. For example, deep learning methods are used to screen pneumonia caused due to COVID-19 (103). Majority voting based classifier ensemble methods and deep CNNs have been used in the diagnosis of COVID-19 from chest X-ray images (104) and CT images (105) respectively. Furthermore, these techniques have been quite successful in the diagnosis of chronic diseases. For example, ensemble methods were used to diagnose coronary heart disease (106-108). An ensemble of deep CNNs were used in classifying interstitial lung abnormality patterns (109). Multi-information fusion neural networks have been applied for the diagnosis of arrhythmia (110). Acute myeloid leukemia (AML) disease has been diagnosed using high performance deep learning methodologies (111).
A weighted decision approach was utilized to diagnose celiac disease (112). Epidemiological predictive model was presented for Bayesian-based pediatric diarrheal disease, which is the second leading cause of mortality in children under 5 years (113).

The decision-making process must be implemented with sufficient accuracy to avoid possible errors and improve the overall quality of healthcare (114). Also, the importance of group decision making in medicine should not be overlooked, since in many cases, this will help clinicians make optimal decisions for their patients (115). A decision-making system based on the fusion of important information is more efficient and robust (116). It provides a shared system to the healthcare providers, enhances the quality of healthcare services and reduces the cost of healthcare for organizations and patients (114).

\section{Applications of DF in the healthcare field}

The applications of the DF technique along with their advantages and disadvantages are presented in four categories: prevention, diagnosis, treatment and administrative. Figure 2 provides a schematic representation of applications of DF in the healthcare field. 


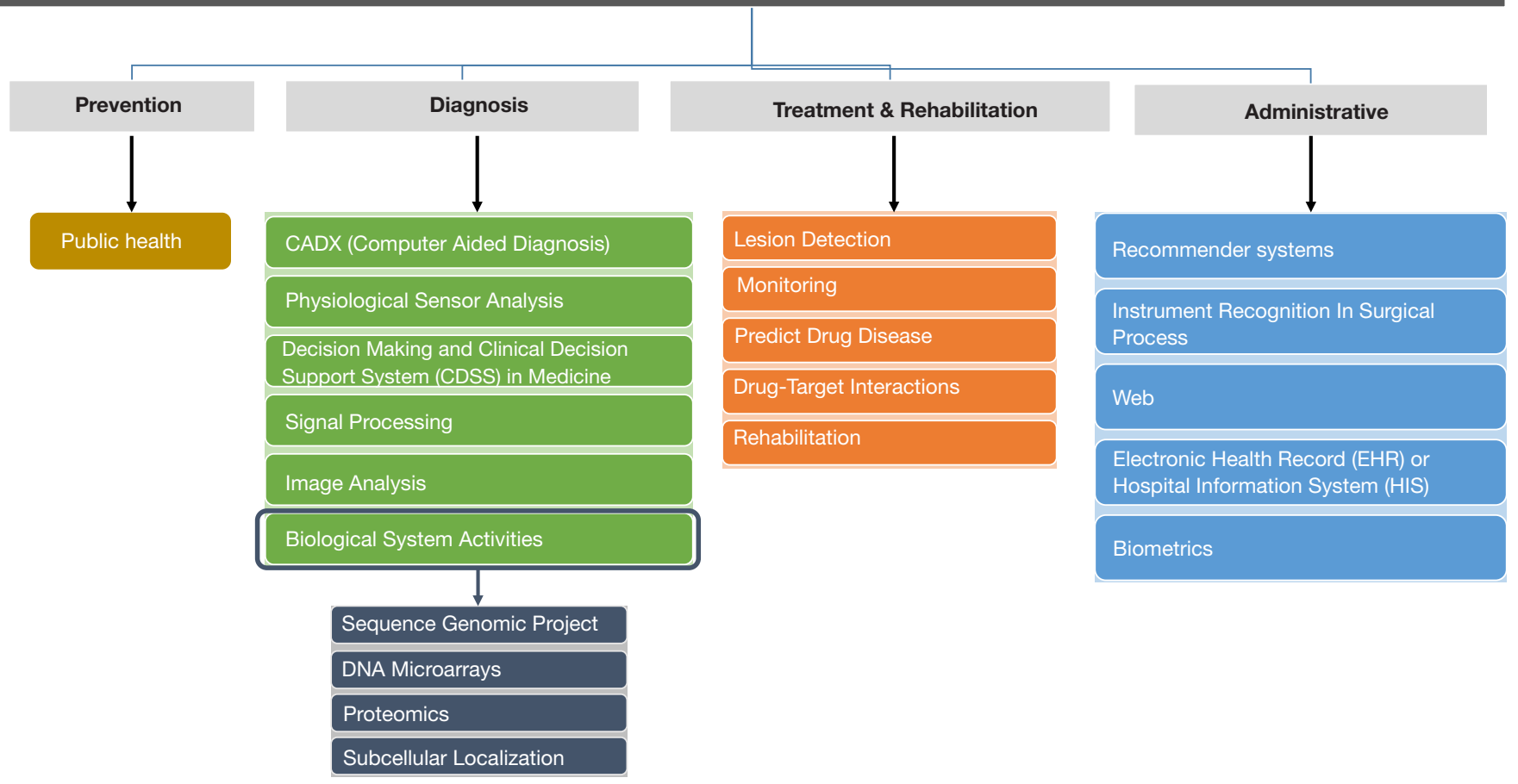

Figure 2 Applications of decision fusion technique according to levels of healthcare delivery.

\section{Prevention level}

DF techniques can be applied at the prevention level to identify the prevalence and incidence of different diseases in heterogeneous populations and to prevent future illnesses. As there is a growing need to merge and share clinical information for decision making between specialties and organizations, DF techniques can be useful. BioPHusion is a program that has been created to support public health surveillance by collecting, monitoring, and synthesizing all types of distributed health information (117).

\section{Diagnosis level}

DF techniques can improve medical diagnosis using computer systems and Clinical Decision Support System (CDSS) in image processing, signal processing, predicting drug efficacy, biological system functions and lesion detection.

\section{The use of DF in computer-aided diagnosis (CAD)}

In recent decades, the research on CAD has rapidly evolved (118). The two most important tasks of CAD are to extract and combine relevant information and their features with other sources to provide reliable diagnosis automatically or semi-automatically (119).
Therefore, CAD approaches are based on their characteristics and classification extractions (109). DF methods play an important role in the application of CAD technology. In one study, the performance of CADbased automatic diabetic retinopathy screening system was evaluated (118). In many CAD approaches, patient images provide different diagnostic outputs, and each image attributes were extracted and analyzed by outputs of the combined classifiers. Liu et al. used 8 attributes for the investigation of microcalcifications on mammograms (120). CAD technology was used to detect benign and malignant breast tumors in several studies $(19,23,36,52,72)$. Other investigators used the Ensemble method for pattern classification and obtained good results (12). These techniques were used for the diagnosis of uterine and cervical cancer histology (12), female pelvic floor lesions (96), small bowel diseases (121-123), central nervous system diseases and prostate cancer (101). Furthermore, CAD can be a very effective tool in geographic areas with low skilled workers. For example, a CAD system was used to differentiate between malignant and benign pigmented skin lesions using lesion boundary detection and quantification of degree of symmetry (124). Because of the fusion of 
various modalities in the $\mathrm{CAD}$ system, providers can now effectively interpret signs and symptoms, thereby, reducing misdiagnosis. Therefore, this field has become a hot topic in the current medical literature (36).

\section{The use of DF for physiological sensor analysis}

Physiological sensor signal analysis is a common practice in medicine to diagnose and classify different physiological conditions. Clinicians frequently use a physiological sensor signal to identify an individual's psychophysiological parameters such as stress, tiredness, fatigue, heart rate, blood pressure and skin temperature. Analyzing data from a single sensor could lead to misleading results (70). Hence, it is necessary to use techniques that manage these diverse data (13), and sensor signal fusion may offer reliable and efficient outcomes (70). In one study, physiological parameters were used to measure stress (70). In another study, the Bayesian network and physiological features were used to detect driver fatigue (125). Other researchers used several sensors to estimate the amount of blood glucose with accuracy in diabetes management (66). In another study, sensor fusion-based methods were used for evaluating physical activity in humans $(62,75)$.

The use of DF for decision-making and CDSS in medicine Decision-making means selecting from several options or creating a solution from a set of options. Fusion techniques can be used to evaluate the options and choosing the best solution from them. For example, when a buyer receives several offers and wants to choose the best one, they need to consider the price, quality and other factors, which is modeled in terms of "multi-preferences" (utility function), also known as group decision-making (GDM) (38). In this decision-making process, the criteria for each option will be individually considered and finally, an optimal decision will be made. Because decision-making plays an important role in healthcare, using these techniques for decision-making can be very helpful. A specialized area within clinical decision-making is the creation of CDSS systems that assists healthcare providers to make prudent judgements. The most recent use of CDSS is the diagnosis of cardiovascular disease. In a study, a series of heterogeneous classifiers including SVM, Bayesian network and artificial neural network (ANN) were used to diagnose cardiovascular disease (126). In another study, three ANNs were used to predict coronary artery disease (127). Neural network ensembles were used to diagnose valvular heart disease (defective function or abnormality of one or more cardiac valves) and satisfactory results have been reported (128).

Given the overarching goal of CDSS is to provide specific recommendations to clinicians for effective decision-making, rather than produce generalizable knowledge, it is not subject to federal regulation (129). It is also not considered a medical device, therefore, does not undergo FDA regulations for medical devices (129). However, it would be argued that considering these systems could result in increased risk of patient harm, it should undergo increased regulation and oversight (129).

\section{The use of DF for signal processing}

Signal processing is another field in which DF techniques can be used to aggregate signals from different sources and choose the best option from them. In a study to classify true or false alarms in the intensive care unit (ICU), these techniques were used for five life-threatening arrhythmias (80). Moreover, this technique was used to analyze cardiorespiratory signals (98), detect epilepsy in neonates (130) and diagnose breast cancer using microwave analysis $(19,131)$.

\section{The use of DF in Image analysis}

By increasing the number, heterogeneity of information resources and data mining techniques especially in complex domains such as image analysis, demand for combining information processes is enhanced to extract valuable knowledge and to make better decisions (36).

With the rise in the number of heterogenous information sources, especially in complex domains such as image analysis, has resulted in an increasing demand for combined information processes to extract valuable information and make better decisions (36). The term DF is used for merging of information from various sources that is done automatically (72). DF is increasingly known as biomedical image analysis. For example, it has been used in CAD and image segmentation (132). Automated segmentation of medical images is a prerequisite for many clinical applications, such as diabetes screening, pancreatic cancer diagnostics and surgical planning. In a study, pancreas segmentation in magnetic resonance imaging (MRI) scans was formulated as a graph-based DF process using deep learning CNNs (76). State of the art in image enhancement and segmentation can now accurately identify" regions of interest" for feature extraction in image enhancement and segmentation (For example, classifying benign versus malignant mammogram images) (72). Also, in some studies, MRI images of the brain were automatically segmented by these techniques. A segmentation study based on these techniques were used to determine the dose painting radiotherapy in positron emission tomography (PET) images $(33,45)$. By using the fusion imaging technique, 
lesion detectability can be augmented, thereby, reducing false positive detection of focal hepatic lesions with a weak ultrasound resolution of two different modalities. In the field of hepatic intervention, real-time ultrasound is typically fused with other imaging modalities such as computed tomography (CT), magnetic resonance (MR) and PET/ $\mathrm{CT}$. These techniques have gained significant popularity to conduct intervention procedures for target lesions with high accuracy and great precision (133). In one study, a complete fusion formulation was proposed in the information space for robust shape tracking, optimally resolving uncertainties in the dynamic system, heteroscedastic measurement noise and subspace shape model in real-time myocardial border tracking for echocardiography sequences (83). In addition to the applications discussed so far, it is widely accepted that applying multiple fields for image data classification, such as images from the MRI, have proven helpful for the early diagnosis of degenerative diseases such as Alzheimer's disease. In a sample of studies, variation of Adaboost methods have been used for this diagnostic purpose (134). In other studies, Classifier Ensembles have been used for the classification of functional magnetic resonance imaging ( $\mathrm{fMRI}$ ) data and is used to display the $3 \mathrm{D}$ structure of the brain with promising results $(31,135-137)$. Some studies have also been conducted on image analysis and computer vision (137). For instance, data from electroencephalogram (EEG) were used for early diagnosis of epilepsy in newborn infants (137).

\section{The use of DF in biological system activities}

The complexity of cell biology is one of the major diagnostic barriers. To cope with this barrier, much attention has been paid to the mathematical modeling of the biological system to understand the molecular structure and their related functions by combining computational, theoretical and experimental techniques (84). Therefore, understanding the internal network of a given system is the ultimate goal in biological studies. While inferring genetic regulatory networks, microarray expression profile data are widely used to examine intracellular activity and a large number of studies have applied computational algorithms to network inference of such data (138). To complete these processes, a large amount of data from diverse sources and algorithms for network inference are needed. Most studies of inferring biological networks have computational and statistical approaches. The Bayesian network is widely used due to a simple mathematical foundation and noise-resistant properties (138). Because of high-throughput technologies, biological system studies and large-scale analysis have expanded from focusing on a single component (e.g., genes) into a large set of components (e.g., whole genome) with the aim of clarifying their interdependencies in diverse biological processes. In addition, when data is imported from different databases, there is a need for integrated analysis to obtain knowledge about the biological system. The aim of biological system activity is to understand the behavior and interactions between various components of the living cell, such as genes, proteins and metabolites. Along with high throughput data, valuable information can be obtained from small-scale experiments, curated databases and computational predictions. Further, dimensionality from the interpretation of heterogeneous data can be reduced by data integration and fusion. In summary, the three main goals are (I) to use heterogeneous data to try and understand the networks that describe biological processes, (II) to attempt to understand the role of genes and proteins in biological processes and how they interact with specific functions and (III) retrieve diverse data from large databases. More recently, many tools have been created to retrieve data from a variety of sources and to integrate and fuse different kinds of genomic data by automatic and semiautomatic methods $(13,138)$.

\section{Genomic sequence project}

Genomic sequence projects and high-speed throughput technologies such as DNA and Protein arrays generate a large amount of rich information. Microarray test data is valuable, but it is a limited source for inferring gene regulation mechanisms at a genomic scale (64). Additional information, such as promoter sequences of genes/DNA binding motifs, gene ontologies and location data can be statistically significant when they are combined with the analysis of the gene expression (64). In one study, machine learning approach was used to combine heterogeneous genomic data. The algorithm used an unsupervised joint learning mechanism to identify gene clusters while using combined data. The results showed that combined learning approaches effectively identify gene expression levels. The algorithm provided an automatic clustering method and allowed the users to determine the influence of each data type on the final clustering through the use of probabilities (64). Despite extensive studies, our understanding of the most genes mechanisms is not complete. Lots of genomic sequencing and expression data allows large-scale studies to be carried out in understanding the mechanisms under gene transcription and regulation control. Accordingly, identifying the transcription factor binding sites (TFBS) is so important. Several algorithms 
have been developed for detecting statistically significant inter-sequence patterns (68).

\section{DNA microarrays}

DNA microarrays are high-throughput experiments that can measure changes in the expression of several thousand of genes simultaneously. The studies of sequence data combined with gene expression were recommended based on the idea that genes with similar shaped expression profiles are likely to be co-regulated and therefore, similar sequence elements may be shared in their promoter areas $(22,139)$. These methods can be broadly divided into two basic categories: the first group of common sequence elements in the promoter regions is searched for genes that have been clustered based on their expression profiles. While the second group finds a group of genes that share similar sequences and then the same expression profiles are searched. In contrast to these methods of making an inference based on a data source, recent methods have been developed to combine gene expression and upstream sequences (68). These techniques were used to fuse DNA and Ribonucleic Acid (RNA) sequences in several applications (38).

\section{Proteomics}

Machine learning techniques are proposed to predict the 3D protein structure. In a study, based on hybrid systems such as ANN, the statistical classifier, and case-based reasoning (CBR) classifier were combined by majority voting and used to predict folds in a study (140). To develop a useful statistical forecasting method for the biological system, we need to consider the following procedure: (I) create or select a valid benchmark for training and testing the model or predictor, (II) formulate statistical samples with an effective mathematical expression that can correctly reflect the internal communication with the target that must be discovered or predicted, (III) introduce or develop a robust algorithm for detection or prediction, (IV) perform crossvalidation tests correctly to maximize predictive accuracy and $(\mathrm{V})$ create a user-friendly web-server for the prediction model or method so that it can be accessible to the public (58).

\section{Subcellular localization}

An important area within bioinformatics is the prediction of subcellular localization of bacterial protein, which is essential for medication formulations and other applications. For these purposes many computing tools have been developed in recent years (141).

Some examples include extraction of sequence data, structural data, tissue expression data and information about the family of proteins (13).

\section{Level of treatment and rehabilitation}

Potential applications of DF techniques at the level of treatment and rehabilitation include detecting the lesion's exact location in surgical procedures, health and disease monitoring, drug efficacy prediction and rehabilitation.

\section{The use of DF for lesion detection}

Surgeons need to have an accurate method to determine the exact location of the lesion. Given that a very small surgical instrument positioning error during the surgery can cause significant damage to the patient, few studies have been conducted to improve the accuracy of neurosurgical procedures (142).

\section{The use of DF for health and disease monitoring}

With the rise in the aging population in developed countries, it becomes imperative to monitor their health trends using approaches such as sensor fusion, thereby reducing the need of human supervision. Fall detection is one such area where body sensors and wireless sensor networks are used to track both physical and mental state of patients. It also attempts to classify an individual state of mind by fusing data from physiological sensors such as heart rate, respiratory rate, carbon dioxide and oxygen level (143). These approaches can also be used to monitor infant gross motor functions with abilities to assess infantile body postures (143). Further these approaches have also been utilized for remote healthcare monitoring (55), tracking of heart rate (89), rehabilitation and knee flexion kinematics (100), differential brain activity classification (90), physical activity $(46,54,66,75,87,90)$ and prosthetic device development using brain computer-interface (BCI) (144).

\section{The use of DF to predict drug efficacy}

Making accurate predictions for drug-disease interactions is a relatively new and challenging field in drug repositioning. Several computational methods use machine learning and network-based approaches to reposition old drugs for new indications. Data fusion methods and aggregation operators are successfully employed to predict unknown drug-disease interactions with greater reliability and precision (145).

\section{The use of DF for drug-target interactions}

It is also used to predict potential targets for a new drug and can address the limitations of multi-target drug and multidrug target studies (146).

\section{The use of DF in rehabilitation}

This technique is widely used to estimate muscle fatigue, predict surface electromyographic muscle movement (sEMG) and detect motion during clinical rehabilitation. 
However, real-time performance and precision of motion decoding based on sEMG signals still pose a challenge. The neural network, fuzzy clustering, wavelet packet, fractal and independent component analysis are some of the special methods used to extract information from non-stationary sEMGs. A personalized system is proposed for the early rehabilitation of post-stroke disabilities (21). Multisource DF techniques have been used for progressive rehabilitation based on real-time evaluation (21). Furthermore, in an information aggregation study of sensors in lower limbs, this technique was used for rehabilitation purposes (100).

\section{Administrative level}

Applications of DF technique at the administrative level include but are not limited to the design of advisory systems for tracking of diseases, proper instrument detection in surgical procedures, exploiting of the webspace for disease management, using EHR and HIS capabilities to improve the quality of care services and biometrics.

\section{The use of DF in recommender systems}

Nowadays, these systems form the basis of a number of researches. It aggregates information to assist customers in choosing their favorite products based on previous searches and transactions. The use of advisory systems in medicine has become increasingly popular. For example, using DF techniques in diabetic drug advocacy systems $(30,140)$.

\section{The use of DF for instrument recognition in the surgical process}

DF techniques have been successfully employed for online recognition of surgical systems (86). In one study, a recognition system was developed by using radio frequency identification (RFID) technology to identify surgical activities. Here, specific sensors were used to abstract and summarize the information. This technique has been able to effectively detect, identify, and localize surgical instruments. The development of automatic situation recognition for surgical process models is a relatively new research field (86). The use of DF in web

Given the dynamic nature of data available on the web, use of DF techniques within this space is of paramount importance (147). Social media is one of the most representative and most relevant sources of big data. Social media data comes from a large number of internet applications and websites, such as Facebook, Twitter, LinkedIn, YouTube, Instagram, Google, Tumblr, Flickr and WordPress. The rapid growth of these websites has allowed users to share and exchange data (148) on these sites, and information is generated from a variety of areas such as e-commerce, e-tourism, hobbies, friendship, education and health. Advertisers can now combine information from different sources and predict user preferences which is often used for marketing purposes. For example, recommender systems can be used to predict user preferences based on search data, and recommendations can be made to increase user engagement and personalization. Social networking sites such as Twitter, Facebook and Flickr also play an important role in disseminating information on current events such as natural disasters, terrorist attacks and other events (149). Platforms such as Twitter have paid careful attention to certain diseases such as influenza (149). Social media platforms have been used effectively for purposes such as self-care, mental health, health information seeking, smoking cessation, improving treatment decision and behavior change (148,150-153). It is possible to process large amount of data from these sites using mathematical techniques such as DS theory and Modified Dempster's rule of combinator to extract useful information. An important threat within the cyberspace is security or privacy of information $(149,154)$. However, DF techniques such as feature extraction using Fisher's linear discriminant analysis and fusion algorithm have been successfully employed to reduce spyware attacks, thereby, guaranteeing high quality secure data (44).

\section{The use of DF in EHR or health information system}

The EHR and HIS are a rich source of patient data including demographic, diagnostic and financial data. This massive amount of data can be used for the improvement of clinical therapeutics, quality of care services and healthcare cost reduction. In a university hospital, data from more than 1,000 patients resulted in more than 1 Gigabyte (GB) of numerical data and laboratory tests in the HIS. The features of this temporary database include: (I) inhomogeneity of each record (according to time-series, including short-term and long-term effects), (II) large number of attributes per record (each record consists of more than 100 attributes), (III) measurement bias for patients suffering from severe chronic illness (large amounts of data stores in a short period). Here, advanced data mining techniques were used to discover useful patterns (12). In one study, a combination of extended-moving, multiscale matching and rule induction method was introduced for discovering new knowledge in medical temporal database and tested in two data sets (12). The results showed desirable gain in knowledge (12). In another study, this technique was used to improve the coding results from various HIS applications and good results were reported (155). Therefore, new 
computational approaches are used widely in the healthcare area since many imprecise and ambiguous data are generated in different sections. Moreover, the range of these applications are very diverse (140).

\section{The use of DF in biometrics}

Biometrics refers to technologies in which behavioral or physiological characteristics are evaluated. These features are unique to each person and can be used to analyze or identify the person. Biometric systems automatically identify individual identities based on physical attributes such as fingerprints, veins, iris, and faces $(44,156)$. A multimodal biometrics system is a relatively new approach that is one of the sub field within the field of information science, which more closely relates to the understanding of adjectives, precise methods and the display of reliable and matching information (44). This domain uses several physiological features for enrollment, verification, and identification to overcome the unimodal biometric problems including interclass variability, interclass similarity, data quality, nonuniversality, sensitivity to noise and other factors to improve performance of the biometric system and recognition rate (44). It has been shown that biometric multimodal systems are more efficient and reliable than unimodal systems. Therefore, the effective fusion scheme is very important for multiple biometric traits $(44,156)$. It is highly recommended to take the advantage of the modal biometric technology as well as recognition using the DF technique in the field of healthcare in the future (99). Furthermore, the use of this technique can be helpful in the field of visual tracking, computer vision and healthcare robotics.

\section{Summary}

The analysis of the massive amounts of data produced in the healthcare sector is necessary to reduce the costs of healthcare, enhance clinical decision-making and improve the quality of healthcare service. DF is one of the big data analysis techniques that have applications at all levels of healthcare delivery, including prevention, diagnosis, treatment, and administration. This technique has been successfully employed in areas such as lesion detection, genomics, cancer diagnosis, image analysis and drugdisease interaction. Intelligent methods such as DF are widely used in healthcare because of capability of this technique to handle complex data within the healthcare environment. Some of the advantages of this technique include good management of non-identical data sources (32), reduce dimensionality, decrease noise (33), improve model performance $(34,35)$ and enhance the interpretation of the results (36). Certain challenges include low quality of data used for analysis, complexity of the data $(40,41)$, selecting the appropriate methods depending on the type of data and computational burden, which should be considered in future studies for achieving better results. Finally, it is recommended to know the advantages, challenges and applications of the technique before its use. Future studies should focus on describing the methodology and types of data used for its applications within the healthcare sector.

\section{Clinical implications}

DF techniques have demonstrated value for diagnosis of various diseases including skeletal, eye and brain diseases. The technique is also recommended in precision medicine to provide accurate diagnosis and treatment recommendations. For the healthcare system, DF can also provide cost savings. The HIS and EHR offer rich data sources that can be manipulated using this technique to improve the quality of care. This technique has proven successful within hospital resource management as well as in admission discharge services.

\section{Acknowledgements}

Funding: This study is the result of a research grant approved by the vice chancellor of research at Mashhad University of Medical Sciences (grant number 961731).

\section{Footnote}

Reporting Checklist: The authors have completed the Narrative Review reporting checklist. Available at https:// dx.doi.org/10.21037/mhealth-21-15

Peer Review File: Available at https://dx.doi.org/10.21037/ mhealth-21-15

Competing Interests: All authors have completed the ICMJE uniform disclosure form (available at https://dx.doi. org/10.21037/mhealth-21-15). EN reports that this study is the result of a research grant approved by the vice chancellor of research at Mashhad University of Medical Sciences (grant number 961731). RB is the co-chair for the Society of Behavioral Medicine (SBM) Women's Health Special Interest Group (SIG)- unpaid. The other authors 
have no conflicts of interest to declare.

Ethical Statement: The authors are accountable for all aspects of the work in ensuring that questions related to the accuracy or integrity of any part of the work are appropriately investigated and resolved.

Open Access Statement: This is an Open Access article distributed in accordance with the Creative Commons Attribution-NonCommercial-NoDerivs 4.0 International License (CC BY-NC-ND 4.0), which permits the noncommercial replication and distribution of the article with the strict proviso that no changes or edits are made and the original work is properly cited (including links to both the formal publication through the relevant DOI and the license). See: https://creativecommons.org/licenses/by-nc-nd/4.0/.

\section{References}

1. Nambiar R, Bhardwaj R, Sethi A, et al. A look at challenges and opportunities of big data analytics in healthcare. 2013 IEEE international conference on Big Data 2013. doi: 10.1109/BigData.2013.6691753

2. Zhang Q, Yang LT, Chen Z, et al. A survey on deep learning for big data. Information Fusion 2018;42:146-57.

3. Raghupathi W, Raghupathi V. Big data analytics in healthcare: promise and potential. Health Inf Sci Syst 2014;2:3.

4. Hekler EB, Klasnja P, Chevance G, et al. Why we need a small data paradigm. BMC Med 2019;17:133.

5. Chen M, Mao S, Liu Y. Big data: A survey. Mob Netw Appl 2014;19:171-209.

6. Murdoch TB, Detsky AS. The inevitable application of big data to health care. JAMA 2013;309:1351-2.

7. Bossé É, Roy J, Wark S, et al. Concepts, Models, and Tools for Information Fusion. Artech House Intelligence and Information Operations Library, Artech House. Inc; 2007.

8. Khan N, Yaqoob I, Hashem IA, et al. Big data: survey, technologies, opportunities, and challenges. ScientificWorldJournal 2014;2014:712826.

9. Jin X, Wah BW, Cheng X, et al. Significance and challenges of big data research. Big Data Res 2015;2:59-64.

10. Esteban J, Starr A, Willetts R, et al. A review of data fusion models and architectures: towards engineering guidelines. Neural Comput Appl 2005;14:273-81.

11. Castanedo F. A review of data fusion techniques. ScientificWorldJournal 2013;2013:704504.
12. Torra V. Information fusion-methods and aggregation operators. Data Mining and Knowledge Discovery Handbook. Boston: Springer; 2009:999-1008.

13. Synnergren J, Olsson B, Gamalielsson J. Classification of information fusion methods in systems biology. In Silico Biol 2009;9:65-76.

14. Balazs JA, Velásquez JD. Opinion mining and information fusion: a survey. Information Fusion 2016;27:95-110.

15. Solaiman B, Debon R, Pipelier F, et al. Information fusion: application to data and model fusion for ultrasound image segmentation. IEEE Trans Biomed Eng 1999;46:1171-5.

16. Mangai UG, Samanta S, Das S, et al. A survey of decision fusion and feature fusion strategies for pattern classification. IETE Technical Review 2010;27:293-307.

17. Terveen LG, Hill W. Beyond recommender systems: Helping people help each other. HCI in the New Millennium 2001;1:487-509.

18. Gunay O, Toreyin BU, Kose K, et al. Entropy-functionalbased online adaptive decision fusion framework with application to wildfire detection in video. IEEE Trans Image Process 2012;21:2853-65.

19. Li Y, Porter E, Santorelli A, et al. Microwave breast cancer detection via cost-sensitive ensemble classifiers: Phantom and patient investigation. Biomed Signal Process Control 2017;31:366-76.

20. Guo P, Banerjee K, Joe Stanley R, et al. Nuclei-Based Features for Uterine Cervical Cancer Histology Image Analysis With Fusion-Based Classification. IEEE J Biomed Health Inform 2016;20:1595-607.

21. Fan Y, Yin Y. Active and Progressive Exoskeleton Rehabilitation Using Multisource Information Fusion From EMG and Force-Position EPP. IEEE Trans Biomed Eng 2013;60:3314-21.

22. Bussemaker HJ, Li H, Siggia ED. Regulatory element detection using correlation with expression. Nat Genet 2001;27:167-71.

23. Nazari E, Chang H-CH, Deldar K et al. A comprehensive overview of decision fusion technique in healthcare: A systematic scoping review. Iran Red Crescent Med J 2020;22:e30.

24. Bello-Orgaz G, Jung JJ, Camacho D. Social big data: Recent achievements and new challenges. Inf Fusion 2016;28:45-59.

25. Moher D, Liberati A, Tetzlaff J, et al. Preferred reporting items for systematic reviews and meta-analyses: the PRISMA statement. BMJ 2009;339:b2535.

26. Kokar MM, Tomasik JA, Weyman J. Data vs. decision fusion in the category theory framework. Proceedings 
of FUSION 2001 - 4th International Conference on Information Fusion, 2001:3-15.

27. Hall DL, Llinas JL. An introduction to multisensory data fusion. Proceedings of the IEEE 1997;85:6-23.

28. Durrant-Whyte HF. Sensor models and multisensory integration. Int J Rob Res 1988;7:97-113.

29. Luo RC, Yih CC, Su KL. Multisensor fusion and integration: approaches, applications, and future research directions. IEEE Sens J 2002;2:107-119.

30. Mei J, Liu H, Li X, et al. A Decision Fusion Framework for Treatment Recommendation Systems. Stud Health Technol Inform 2015;216:300-4.

31. Sakkalis V, Zervakis M, Micheloyannis S. Biopattern initiative: towards the development and integration of next-generation information fusion approaches. The 26th Annual International Conference of the IEEE Engineering in Medicine and Biology Society, 2004:399-402.

32. Fontana JM, Farooq M, Sazonov E. Automatic ingestion monitor: a novel wearable device for monitoring of ingestive behavior. IEEE Trans Biomed Eng 2014;61:1772-9.

33. Lelandais B, Ruan S, Denœux T, et al. Fusion of multitracer PET images for dose painting. Med Image Anal 2014;18:1247-59.

34. Acharya S, Rajasekar A, Shender BS, et al. Real-Time Hypoxia Prediction Using Decision Fusion. IEEE J Biomed Health Inform 2017;21:696-707.

35. O'Regan S, Marnane W. Multimodal detection of headmovement artefacts in EEG. J Neurosci Methods 2013;218:110-20.

36. Velikova M, Lucas PJ, Samulski M, et al. A probabilistic framework for image information fusion with an application to mammographic analysis. Med Image Anal 2012;16:865-75.

37. Bossé É, Solaiman B. Information fusion and analytics for big data and IoT. Artech House; 2016.

38. Torra V, Narukawa Y. Modeling decisions: information fusion and aggregation operators. Springer Science \& Business Media; 2007.

39. Zhong H, Xiao J. Enhancing health risk prediction with deep learning on big data and revised fusion node paradigm. Scientific Programming 2017. doi: 10.1155/2017/1901876

40. Liu Y'T, Pal NR, Marathe AR, et al. Fuzzy DecisionMaking Fuser (FDMF) for Integrating Human-Machine Autonomous (HMA) Systems with Adaptive Evidence Sources. Front Neurosci 2017;11:332.

41. Anderson F, Birch DW, Boulanger P, et al. Sensor fusion for laparoscopic surgery skill acquisition. Comput Aided Surg 2012;17:269-83.

42. Fouad MM, Oweis NE, Gaber T, et al. Data mining and fusion techniques for WSNs as a source of the big data. Procedia Comput Sci 2015;65:778-86.

43. Nazari E, Pour R, Tabesh H. Comprehensive overview of decision-fusion technique in healthcare: A scoping review protocol. Frontiers in Health Informatics 2018;7:e7.

44. Paul PP, Gavrilova ML, Alhajj R. Decision fusion for multimodal biometrics using social network analysis. IEEE Trans Syst Man Cybern Syst 2014;44:1522-33.

45. Lelandais B, Gardin I, Mouchard L, et al. Segmentation of biological target volumes on multi-tracer PET images based on information fusion for achieving dose painting in radiotherapy. Med Image Comput Comput Assist Interv 2012;15:545-52.

46. Kook H, Gupta L, Kota S, et al. A dynamic multichannel decision-fusion strategy to classify differential brain activity. Annu Int Conf IEEE Eng Med Biol Soc 2007;2007:3212-5.

47. Antink CH, Gao H, Brüser C, et al. Beat-to-beat heart rate estimation fusing multimodal video and sensor data. Biomed Opt Express 2015;6:2895-907.

48. Yue D, Guo M, Chen Y, et al. A Bayesian decision fusion approach for microRNA target prediction. BMC Genomics 2012;13 Suppl 8:S13.

49. Liu F, Zhang SW, Guo WF, et al. Inference of Gene Regulatory Network Based on Local Bayesian Networks. PLoS Comput Biol 2016;12:e1005024.

50. Daunizeau J, Grova C, Marrelec G, et al. Symmetrical event-related EEG/fMRI information fusion in a variational Bayesian framework. Neuroimage 2007;36:69-87.

51. Mnatsakanyan ZR, Burkom HS, Hashemian MR, et al. Distributed information fusion models for regional public health surveillance. Information Fusion 2012;13:129-36.

52. Jesneck JL, Nolte LW, Baker JA, et al. Optimized approach to decision fusion of heterogeneous data for breast cancer diagnosis. Med Phys 2006;33:2945-54.

53. Meng X, Zhang ZQ, Wu JK, et al. Hierarchical information fusion for global displacement estimation in microsensor motion capture. IEEE Trans Biomed Eng 2013;60:2052-63.

54. Qi J, Yang P, Hanneghan M, et al. Multiple density maps information fusion for effectively assessing intensity pattern of lifelogging physical activity. Neurocomputing 2017;220:199-209.

55. Sung WT, Chang KY. Evidence-based multi-sensor 
information fusion for remote health care systems.

Sensors and Actuators A Physical 2013. doi: 10.1016/ j.sna.2013.09.034.

56. Singh R, Murad W. Protein disulfide topology determination through the fusion of mass spectrometric analysis and sequence-based prediction using DempsterShafer theory. BMC Bioinformatics 2013;14 Suppl 2:S20.

57. Wang J, Hu Y, Xiao F, et al. A novel method to use fuzzy soft sets in decision making based on ambiguity measure and Dempster-Shafer theory of evidence: An application in medical diagnosis. Artif Intell Med 2016;69:1-11.

58. Zhang SW, Zhang TH, Zhang JN, et al. Prediction of Signal Peptide Cleavage Sites with Subsite-Coupled and Template Matching Fusion Algorithm. Mol Inform 2014;33:230-9.

59. Yang $\mathrm{P}, \mathrm{Xu} \mathrm{L}$, Zhou BB, et al. A particle swarm based hybrid system for imbalanced medical data sampling. BMC Genomics 2009;10 Suppl 3:S34.

60. Barra V, Boire JY. Automatic segmentation of subcortical brain structures in MR images using information fusion. IEEE Trans Med Imaging 2001;20:549-58.

61. Depeursinge A, Racoceanu D, Iavindrasana J, et al. Fusing visual and clinical information for lung tissue classification in high-resolution computed tomography. Artif Intell Med 2010;50:13-21.

62. Liu S, Gao RX, John D, Staudenmayer J, Freedson PS. SVM-based multi-sensor fusion for free-living physical activity assessment. Annu Int Conf IEEE Eng Med Biol Soc 2011;2011:3188-91.

63. Re M, Valentini G. Integration of heterogeneous data sources for gene function prediction using decision templates and ensembles of learning machines. Neurocomputing 2010;73:1533-7.

64. Kasturi J, Acharya R. Clustering of diverse genomic data using information fusion. Bioinformatics 2005;21:423-9.

65. Wei J, Chan HP, Zhou C, et al. Computer-aided detection of breast masses: four-view strategy for screening mammography. Med Phys 2011;38:1867-76.

66. Xiong F, Hipszer BR, Joseph J, et al. Improved blood glucose estimation through multi-sensor fusion. Annu Int Conf IEEE Eng Med Biol Soc 2011;2011:377-80.

67. Zhang YC, Zhang SW, Liu L, et al. Spatially Enhanced Differential RNA Methylation Analysis from AffinityBased Sequencing Data with Hidden Markov Model. Biomed Res Int 2015;2015:852070.

68. Haase S, Forman C, Kilgus T, et al. ToF/RGB sensor fusion for 3-D endoscopy. Curr Med Imaging 2013;9:113-9.
69. Liu H, Shi X, Guo D, et al. Feature Selection Combined with Neural Network Structure Optimization for HIV1 Protease Cleavage Site Prediction. Biomed Res Int 2015;2015:263586.

70. Barua S. Multi-sensor information fusion for classification of driver's physiological sensor data. [master's thesis on the Internet]. Västerås, Sweden: Mälardalen University; 2013.

71. Ponti MP Jr. Combining classifiers: from the creation of ensembles to the decision fusion. 2011 24th SIBGRAPI Conference on Graphics, Patterns, and Images Tutorials, 2011:1-10.

72. Prasad S, Bruce LM, Ball JE. A multi-classifier and decision fusion framework for robust classification of mammographic masses. Annu Int Conf IEEE Eng Med Biol Soc 2008;2008:3048-51.

73. Ahiskali M, Green D, Kounios J, et al. ERP based decision fusion for AD diagnosis across cohorts. Annu Int Conf IEEE Eng Med Biol Soc 2009;2009:2494-7.

74. Stroud J, Enverga I, Silverstein T, et al. Ensemble learning and the heritage health prize. Irvine, CA: University of California; 2012.

75. Chowdhury AK, Tjondronegoro D, Chandran V, et al. Ensemble Methods for Classification of Physical Activities from Wrist Accelerometry. Med Sci Sports Exerc 2017;49:1965-73.

76. Cai J, Lu L, Zhang Z, et al. Pancreas segmentation in MRI using graph-based decision fusion on convolutional neural networks. Med Image Comput Comput Assist Interv 2016;9901:442-50.

77. Sert E, Ertekin S, Halici U. Ensemble of convolutional neural networks for classification of breast microcalcification from mammograms. Annu Int Conf IEEE Eng Med Biol Soc 2017;2017:689-92.

78. Fan XN, Zhang SW. lncRNA-MFDL: identification of human long non-coding RNAs by fusing multiple features and using deep learning. Mol Biosyst 2015;11:892-7.

79. Kochi N, Helikar T, Allen L, et al. Sensitivity analysis of biological Boolean networks using information fusion based on nonadditive set functions. BMC Syst Biol 2014;8:92.

80. Liu C, Zhao L, Tang H, et al. Life-threatening false alarm rejection in ICU: using the rule-based and multichannel information fusion method. Physiol Meas 2016;37:1298-312.

81. Yanq X, Ye Y, Xia Y, et al. A precise and accurate acupoint location obtained on the face using consistency matrix pointwise fusion method. J Tradit Chin Med 2015;35:110-6. 
82. Mou Q, Xu Z, Liao H. An intuitionistic fuzzy multiplicative best-worst method for multi-criteria group decision making. Information Sciences 2016;374:224-39.

83. Comaniciu D, Zhou XS, Krishnan S. Robust real-time myocardial border tracking for echocardiography: an information fusion approach. IEEE Trans Med Imaging 2004;23:849-60.

84. Kolesar I, Parulek J, Viola I, et al. Interactively illustrating polymerization using three-level model fusion. BMC Bioinformatics 2014;15:345.

85. Chua HN, Sung WK, Wong L. An efficient strategy for extensive integration of diverse biological data for protein function prediction. Bioinformatics 2007;23:3364-73.

86. Neumuth T, Meissner C. Online recognition of surgical instruments by information fusion. Int J Comput Assist Radiol Surg 2012;7:297-304.

87. Chowdhury AK, Tjondronegoro D, Chandran V, et al. Physical Activity Recognition Using Posterior-Adapted Class-Based Fusion of Multiaccelerometer Data. IEEE J Biomed Health Inform 2018;22:678-85.

88. Chen C, Ugon A, Zhang X, et al. Personalized sleep staging system using evolutionary algorithm and symbolic fusion. Annu Int Conf IEEE Eng Med Biol Soc 2016;2016:2266-9.

89. Yang P, Dumont GA, Ansermino JM. Sensor fusion using a hybrid median filter for artifact removal in intraoperative heart rate monitoring. J Clin Monit Comput 2009;23:75-83.

90. Gupta L, Chung B, Srinath MD, et al. Multichannel fusion models for the parametric classification of differential brain activity. IEEE Trans Biomed Eng 2005;52:1869-81.

91. Köhler T, Haase S, Bauer S, et al. Multi-sensor superresolution for hybrid range imaging with application to 3-D endoscopy and open surgery. Med Image Anal 2015;24:220-34.

92. Heckemann RA, Hajnal JV, Aljabar P, et al. Automatic anatomical brain MRI segmentation combining label propagation and decision fusion. Neuroimage 2006;33:115-26.

93. Tahir BA, Swift AJ, Marshall H, et al. A method for quantitative analysis of regional lung ventilation using deformable image registration of CT and hybrid hyperpolarized gas/1H MRI. Phys Med Biol 2014;59:7267-77.

94. Mahdavi SS, Moradi M, Morris WJ, et al. Fusion of ultrasound B-mode and vibro-elastography images for automatic 3D segmentation of the prostate. IEEE Trans Med Imaging 2012;31:2073-82.
95. Yang K, Koo HW, Park W, et al. Fusion 3-Dimensional Angiography of Both Internal Carotid Arteries in the Evaluation of Anterior Communicating Artery Aneurysms. World Neurosurg 2017;98:484-91.

96. Akhondi-Asl A, Hoyte L, Lockhart ME, et al. A logarithmic opinion pool based STAPLE algorithm for the fusion of segmentations with associated reliability weights. IEEE Trans Med Imaging 2014;33:1997-2009.

97. Luo X, Wan Y, He X. Robust electromagnetically guided endoscopic procedure using enhanced particle swarm optimization for multimodal information fusion. Med Phys 2015;42:1808-17.

98. Antink CH, Leonhardt S, Walter M. A synthesizer framework for multimodal cardiorespiratory signals. Biomed Phys Eng Express 2017;3:035028.

99. Li X, Dick A, Shen C, et al. Visual tracking with spatiotemporal Dempster-Shafer information fusion. IEEE Trans Image Process 2013;22:3028-40.

100. Tannous H, Istrate D, Benlarbi-Delai A, et al. A New Multi-Sensor Fusion Scheme to Improve the Accuracy of Knee Flexion Kinematics for Functional Rehabilitation Movements. Sensors (Basel) 2016. doi: 10.3390/ s16111914.

101. Tiwari P, Viswanath S, Kurhanewicz J, et al. Multimodal wavelet embedding representation for data combination (MaWERiC): integrating magnetic resonance imaging and spectroscopy for prostate cancer detection. NMR Biomed 2012;25:607-19.

102. Nath A, Subbiah K. Maximizing lipocalin prediction through balanced and diversified training set and decision fusion. Comput Biol Chem 2015;59 Pt A:101-10.

103.Xu X, Jiang X, Ma C, et al. A Deep Learning System to Screen Novel Coronavirus Disease 2019 Pneumonia. Engineering (Beijing) 2020;6:1122-9.

104. Chandra TB, Verma K, Singh BK, et al. Coronavirus disease (COVID-19) detection in Chest X-Ray images using majority voting based classifier ensemble. Expert Syst Appl 2021;165:113909.

105. Mishra AK, Das SK, Roy P, et al. Identifying COVID19 from Chest CT Images: A Deep Convolutional Neural Networks Based Approach. J Healthc Eng 2020;2020:8843664.

106. Tama BA, Im S, Lee S. Improving an Intelligent Detection System for Coronary Heart Disease Using a Two-Tier Classifier Ensemble. Biomed Res Int 2020;2020:9816142.

107.Li Y, He Z, Wang H, et al. CraftNet: A deep learning ensemble to diagnose cardiovascular diseases. Biomed Signal Process Control 2020;62:102091. 
108. Nugroho KA, Setiawan NA, Adji TB. Coronary heart disease diagnosis based on improved ensemble learning. Journal of Convergence Information Technology 2013;8:13-24.

109. Bermejo-Peláez D, Ash SY, Washko GR, et al. Classification of Interstitial Lung Abnormality Patterns with an Ensemble of Deep Convolutional Neural Networks. Sci Rep 2020;10:338.

110.Chen A, Wang F, Liu W, et al. Multi-information fusion neural networks for arrhythmia automatic detection. Comput Methods Programs Biomed 2020;193:105479.

111. Nazari E, Farzin AH, Aghemiri M, et al. Deep Learning for Acute Myeloid Leukemia Diagnosis. J Med Life 2020;13:382-7.

112. Gadermayr M, Uhl A, Vécsei A. Quality based information fusion in fully automatized celiac disease diagnosis. German Conference on Pattern Recognition. Springer, Cham, 2014:666-77.

113.Heaney AK, Alexander KA, Shaman J. Ensemble forecast and parameter inference of childhood diarrhea in Chobe District, Botswana. Epidemics 2020;30:100372.

114.Li M, Chapman GB. Medical decision making. The Wiley Encyclopedia of Health Psychology 2020:347-53.

115. Oshima Lee E, Emanuel EJ. Shared decision making to improve care and reduce costs. N Engl J Med 2013;368:6-8.

116. Rahman MM, Bhattacharya P. An integrated and interactive decision support system for automated melanoma recognition of dermoscopic images. Comput Med Imaging Graph 2010;34:479-86.

117. Khan AS, Fleischauer A, Casani J, et al. The next public health revolution: public health information fusion and social networks. Am J Public Health 2010;100:1237-42.

118. Niemeijer M, Abramoff MD, van Ginneken B. Information fusion for diabetic retinopathy CAD in digital color fundus photographs. IEEE Trans Med Imaging 2009;28:775-85.

119. Quellec G, Lamard M, Cazuguel G, et al. Case retrieval in medical databases by fusing heterogeneous information. IEEE Trans Med Imaging 2011;30:108-18.

120.Liu B, Metz CE, Jiang Y. An ROC comparison of four methods of combining information from multiple images of the same patient. Med Phys 2004;31:2552-63.

121.Zhao Q, Mullin GE, Meng MQ, et al. A general framework for wireless capsule endoscopy study synopsis. Comput Med Imaging Graph 2015;41:108-16.

122. Richard N, Dojat M, Garbay C. Automated segmentation of human brain MR images using a multi-agent approach. Artif Intell Med 2004;30:153-75.

123.Zhu C, Jiang T. Multicontext fuzzy clustering for separation of brain tissues in magnetic resonance images. Neuroimage 2003;18:685-96.

124. Schmid-Saugeona P, Guillodb J, Thirana JP. Towards a computer-aided diagnosis system for pigmented skin lesions. Comput Med Imaging Graph 2003;27:65-78.

125. Yang G, Lin Y, Bhattacharya P. A driver fatigue recognition model based on information fusion and dynamic Bayesian network. Information Sciences 2010;180:1942-54.

126.Eom JH, Kim SC, Zhang BT. AptaCDSS-E: A classifier ensemble-based clinical decision support system for cardiovascular disease level prediction. Expert Syst Appl 2008;34:2465-79.

127. Das R, Turkoglu I, Sengur A. Effective diagnosis of heart disease through neural networks ensembles. Expert Syst Appl 2009;36:7675-80.

128. Das R, Turkoglu I, Sengur A. Diagnosis of valvular heart disease through neural networks ensembles. Comput Methods Programs Biomed 2009;93:185-91.

129. Evans EL, Whicher D. What Should Oversight of Clinical Decision Support Systems Look Like? AMA J Ethics 2018;20:E857-863.

130. Malarvili MB, Mesbah M. Combining newborn EEG and HRV information for automatic seizure detection. Annu Int Conf IEEE Eng Med Biol Soc 2008;2008:4756-9.

131. Kirshin E, Oreshkin B, Zhu GK, et al. Microwave radar and microwave-induced thermoacoustics: dual-modality approach for breast cancer detection. IEEE Trans Biomed Eng 2013;60:354-60.

132.Zanaty E. An approach based on fusion concepts for improving brain Magnetic Resonance Images (MRIs) segmentation. J Med Imaging Health Inform 2013;3:30-7.

133. Lee MW. Fusion imaging of real-time ultrasonography with CT or MRI for hepatic intervention. Ultrasonography 2014;33:227-39.

134. Savio A, García-Sebastián MT, Chyzyk D, et al. Neurocognitive disorder detection based on feature vectors extracted from VBM analysis of structural MRI. Comput Biol Med 2011;41:600-10.

135. Kuncheva LI, Rodríguez JJ. Classifier ensembles for fMRI data analysis: an experiment. Magn Reson Imaging 2010;28:583-93.

136.Plumpton CO, Kuncheva LI, Oosterhof NN, et al. Naive random subspace ensemble with linear classifiers for realtime classification of fMRI data. Pattern Recognition 2012;45:2101-8.

137.Li H, Jeremíc A. Neonatal seizure detection using blind distributed detection with correlated decisions. Annu Int Conf IEEE Eng Med Biol Soc 2011;2011:6580-4. 
138. Lee D, Kim S, Kim Y. BioCAD: an information fusion platform for bio-network inference and analysis. BMC Bioinformatics 2007;8 Suppl 9:S2.

139. Holmes I, Bruno WJ. Finding regulatory elements using joint likelihoods for sequence and expression profile data. Proc Int Conf Intell Syst Mol Biol 2000;8:202-10.

140. Wo niak M, Grana M, Corchado E. A survey of multiple classifier systems as hybrid systems. Information Fusion 2014;16:3-17.

141. Chen J, Xu H, He PA, et al. A multiple information fusion method for predicting subcellular locations of two different types of bacterial protein simultaneously. Biosystems 2016;139:37-45

142.Kim DN, Chae YS, Kim MY. X-ray and optical stereo-based 3D sensor fusion system for imageguided neurosurgery. Int J Comput Assist Radiol Surg 2016;11:529-41.

143. Fung ML, Chen MZ, Chen YH. Sensor fusion: A review of methods and applications. 2017 29th Chinese Control And Decision Conference (CCDC), 2017:3853-60.

144. White JR, Levy T, Bishop W, et al. Real-time decision fusion for multimodal neural prosthetic devices. PLoS One 2010;5:e9493.

145.Moghadam H, Rahgozar M, Gharaghani S. Scoring multiple features to predict drug disease associations using information fusion and aggregation. SAR QSAR Environ Res 2016;27:609-28.

146.Peng L, Liao B, Zhu W, et al. Predicting Drug-Target Interactions With Multi-Information Fusion. IEEE J Biomed Health Inform 2017;21:561-72.

147. Yao J, Raghavan VV, Wu Z. Web information fusion:

doi: $10.21037 /$ mhealth-21-15

Cite this article as: Nazari E, Biviji R, Roshandel D, Pour $\mathrm{R}$, Shahriari MH, Mehrabian A, Tabesh H. Decision fusion in healthcare and medicine: a narrative review. mHealth 2022;8:8.
A review of the state of the art. Information Fusion 2008;9:446-9.

148. Keller B, Labrique A, Jain KM, et al. Mind the gap: social media engagement by public health researchers. J Med Internet Res 2014;16:e8.

149.Abirami T, Taghavi E, Tharmarasa R,et al. Fusing social network data with hard data. 2015 18th International Conference on Information Fusion (Fusion), 2015:652-8.

150. Mota Pereira J. Facebook enhances antidepressant pharmacotherapy effects. ScientificWorldJournal 2014;2014:892048.

151. Cobb NK, Jacobs MA, Saul J, et al. Diffusion of an evidence-based smoking cessation intervention through Facebook: a randomised controlled trial study protocol. BMJ Open 2014;4:e004089.

152. Wicks P, Massagli M, Frost J, et al. Sharing health data for better outcomes on PatientsLikeMe. J Med Internet Res 2010;12:e19.

153. Timian A, Rupcic S, Kachnowski S, et al. Do patients "like" good care? measuring hospital quality via Facebook. Am J Med Qual 2013;28:374-82.

154. Gao P, Baras JS, Golbeck J. Semiring-based trust evaluation for information fusion in social network services. 2015 18th International Conference on Information Fusion (Fusion), 2015:590-6.

155.Lecornu L, Le Guillou C, Le Saux F, et al. Information fusion for diagnosis coding support. Annu Int Conf IEEE Eng Med Biol Soc 2011;2011:3176-9.

156. Monwar MM, Gavrilova ML. Multimodal biometric system using rank-level fusion approach. IEEE Trans Syst Man Cybern B Cybern 2009;39:867-78. 